\title{
Intermediate Holographic Data Storage System by Using Sequentially Superimposed Recording
}

\author{
Jong Su Yi and Yeon Ho Lee* \\ Department of Information and Communication Engineering, Sungkyunkwan University, \\ Suwon 440-746, Korea
}

(Received August 27, 2009 : revised November 9, 2009 : accepted November 12, 2009)

\begin{abstract}
We introduce a holographic data storage system for intermediating between small data sets and mass holographic data recording. It employs a holographic sequentially superimposed recording technique. We discuss a time scheduling technique for making uniform reconstruction of sequentially recorded holograms and we show experimental results. We also discuss the Bragg selectivity of sequentially recorded holograms. The maximum storage density of our system is estimated to be $224 \mathrm{kbit} / \mathrm{mm}^{2}$. Our system is useful as an intermediate recording system before recording mass holographic data in a larger system.
\end{abstract}

Keywords: Holographic data storage, Sequentially superimposed holograms, Time scheduling, Bragg selectivity

OCIS codes : (090.0090) Holography; (210.0210) Optical data storage; (090.4220) Multiplex holography; (090.7330) Volume gratings

\section{INTRODUCTION}

Holography is very useful and interesting technology in many optical areas such as optical components [1], display [2, 3], microscopy $[4,5]$ and so on. Also, holographic storage technology is one of the most extensively studied mass storage technologies in recent years because of the extended internet usage, the development of three dimensional display systems, and therefore the great demand for mass storage systems. Holographic storage technology is the most promising candidate.

The advantage of the holographic storage technology comes from the parallel data processing and the hologram multiplexing. In this system a large quantity of data is first arranged in a two dimensional data page and then it is recorded all together during a short period of time. At the data reading process, the two dimensional data is restored instantaneously and at the same time by a single illumination by the reference beam. These make it possible for the holographic data storage systems to have a rapid data transfer rate.

However, the parallel data processing may reduce the system efficiency in some cases. For example, class grades, daily diaries, and short e-mails produce files of small size. If one tries to record these small files in a

*Corresponding author: yeonlee@ece.skku.ac.kr holographic data storage system that is capable of recording a large size data page all at once, the data page may not be filled with these small files and may be recorded with a lot of blank data that will result in system waste. In this case, an intermediate data storage system is needed to reduce the system waste.

In this paper, we propose an intermediate holographic data storage system in which one dimensional data is sequentially recorded and, then, the recorded data is restored simultaneously in a two dimensional data page as in the conventional holographic data storage system.

The system is based on the technique of sequentially superimposed hologram recording. This technique has been widely studied in different approaches. LaMacchia and Vincelette discussed the diffraction efficiency of the sequentially recorded hologram and that of the single hologram [6]. Carlsen showed that sequential bit recording could be used to form a page-oriented hologram memory [7]. Kostuk et. al. used a coupled wave model to analyze both simultaneously and sequentially recorded holograms [8].

In our intermediate holographic data storage system, a one dimensional spatial light modulator is used together with the sequential holographic recording technique. First, the principle of the sequential recording technique is explained. Then, the experimental setup is explained. Also discussed in this section is the time scheduling 
(a)

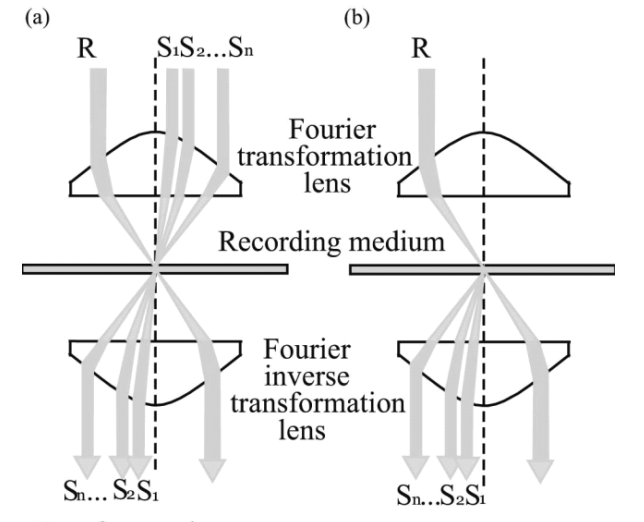

$\mathrm{R}$ : reference beam

$\mathrm{S}_{1}, \mathrm{~S}_{2}, \ldots, \mathrm{S}_{\mathrm{n}}$ : signal beams

FIG. 1. Schematics of data recording and reconstruction. (a) Sequential data recording. (b) Simultaneous reconstruction of data.

technique required for the uniform reconstruction of sequentially recorded signals. Finally, the Bragg selectivity of the sequentially recorded hologram is discussed.

\section{SEQUENTIALLY SUPERIMPOSED HOLOGRAPHIC RECORDING AND EXPERIMENTAL SETUP}

\section{Sequentially superimposed holographic recording}

In the conventional holographic recording method, a two-dimensional data page is imposed to the signal beam and it is recorded by the interference between the signal and the reference beam. However, in our system using a sequentially superimposed holographic recording method, the data page is separated into multiples of one dimensional column data and they are imposed on separate signal beams. Each signal beam interferes with the same reference beam, at different times, and records the data sequentially.

Fig. 1 shows schematics of our system for recording and reconstructing $n$ signal beams. First, as shown in Fig. 1(a), the reference beam $R$ interferes with the $1^{\text {st }}$ signal beam $S_{1}$ and records the data on the recording medium such as photopolymer. Next, the same reference beam interferes with the second signal beam $S_{2}$ to record the second data set, and so on. Fig. 1(b) shows that the recorded $n$ data are reconstructed by the same reference beam at the same time.

\section{Experimental setup}

Fig. 2 shows the experimental setup used in our experiment. The light source is a DPSS laser of $532 \mathrm{~nm}$ wavelength. The laser beam first passes through switch 1 , which is used to control the exposure time during the recording procedure.

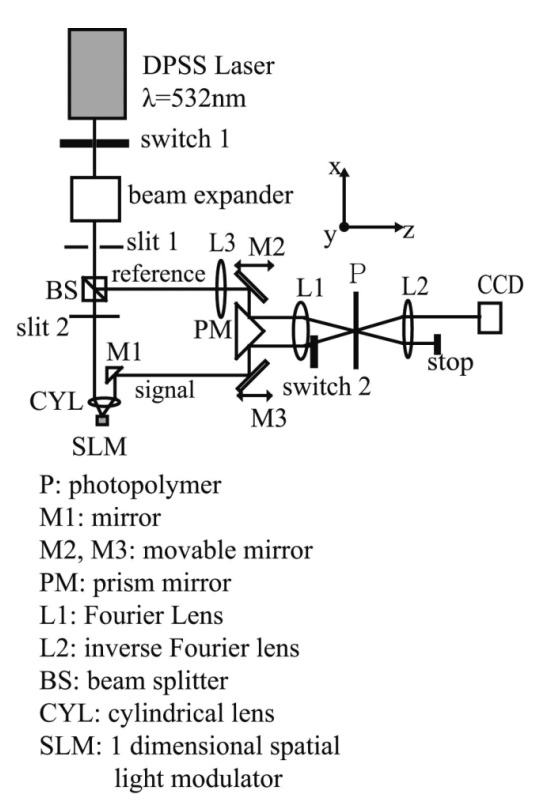

FIG. 2. Setup for holographic superimposed recording.

It is also used to make the reconstructed signal beams be uniform in intensities. The beam is then expanded by a beam expander to a beam of diameter about 3 $\mathrm{cm}$. Slit 1 is used to truncate this light beam and make the beam more uniform in intensity. The beam from slit 1 passes through a non-polarizing beam splitter and forms a signal beam and a reference beam.

The reference beam from the beam splitter passes through lens L3 and is deliberately defocused on the recording medium $\mathrm{P}$. We will discuss the need to defocus in the lens system section in detail. The movable mirror M2 reflects the reference beam such that the beam direction is changed according to its displacement after the lens L1, which is used to make the interference between the reference and the signal beam. In other words, a small movement of the mirror M2 makes the incidence angle of the reference beam change at the recording medium.

Slit 2 is used to truncate the signal beam for the proper light shape to be used in the spatial light modulator. The cylindrical lens CYL focuses the signal beam from slit 2 to the effective area of the spatial light modulator SLM, which will modulate and reflect the signal beam. We will discuss the spatial light modulator in detail in the spatial light modulator section. The modulated signal beam passes through the same cylindrical lens again. It makes the signal beam be a parallel beam. Mirror 3 is movable and it, together with the lens L1, causes the incident angle of the signal beam to change at the recording medium. The incident angle change caused by the position change of mirror 3 makes it possible to separate the signal beams recorded during the sequential recording procedure. The lens L1 is a Fourier lens that also focuses the signal beam from 


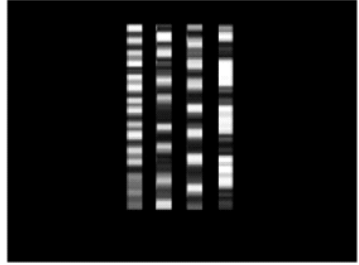

(a)

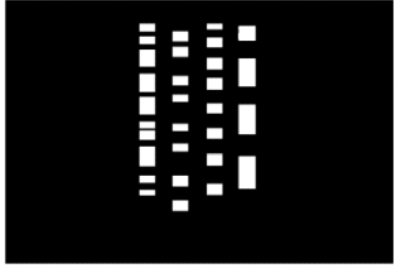

(b)
FIG. 3. The reconstructed signals (a) before digitizing, (b) after digitizing.

the prism mirror on the recording medium. Before it reaches on the recording medium, it passes through switch 2. We open switch 2 during the recording process and close it during the reconstructing process. During the reconstruction process, the lens L2 performs the inverse Fourier transform and restores the image of the signal beam at the CCD plane.

Figure 3 shows an example of the reconstructed image. Fig. 3(a) is the reconstructed original signal beam before digitizing and Fig 3(b) is that after digitizing. The width of slit 2 was about $0.5 \mathrm{~mm}$ and the spatial interval between the mirror movements was $0.75 \mathrm{~mm}$ in our experiment. We recorded four signals in a photo polymer of thickness $100 \mu \mathrm{m}$. In this case pixels of the spatial light modulator were not matched with those of CCD and the contrast of the spatial light modulator was measured as 5.6 because it was operating at the preliminary mode.

The digitizing of the data is done by the threshold value properly selected from the intensity histogram of the reconstructed signals. When the signal is recorded without the time scheduling method, which will be explained in detail in part III, the intensity histogram of the reconstructed signals has a broad distribution so that it may be impossible to find any threshold values. However, when the signal is recorded using the time scheduling method, the intensity histogram has two peaks and the threshold value can be found at the valley between the two peaks.

\section{Lens system}

In recent studies of the holographic storage system, the co-axial lens system has been one of the main issues [9-13]. This lens system is simple and makes it easy to perform the angular multiplexing. Our holographic sequential superimposed recording system consists of two step superimposing processes. One superimposes many signals with one reference beam and the other multiplexes the two dimensional data pages with several reference beams. To utilize the simple mechanical operation, our storage system uses a partially co-axial lens system.

Our lens system is partially co-axial because the lens L3 defocuses the reference beam on the photopolymer

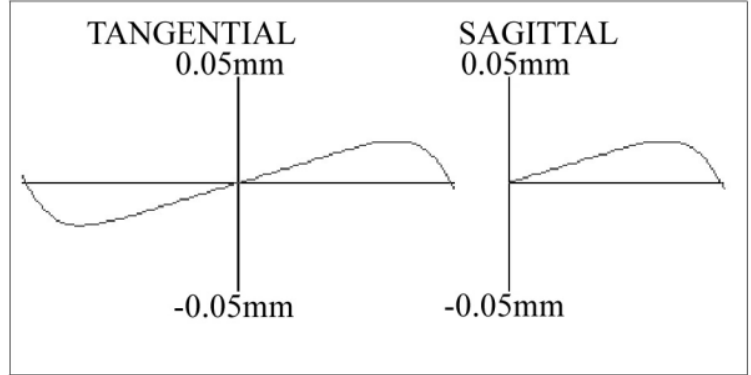

FIG. 4. The ray aberration curves of the Fourier lens in our experiment.

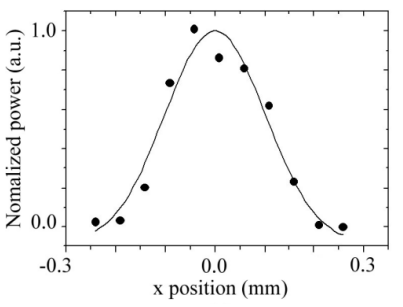

(a)

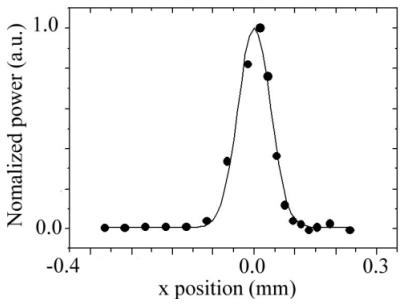

(c)

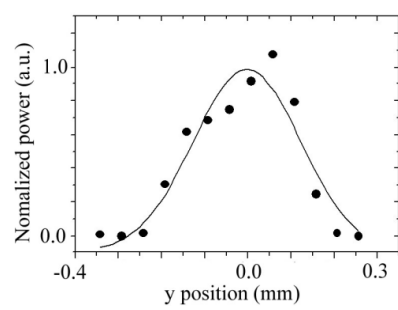

(b)

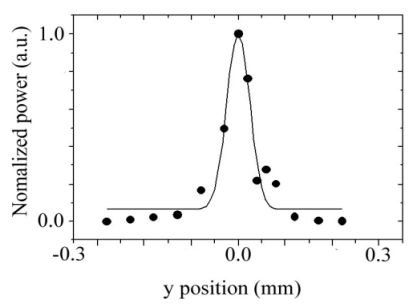

(d)
FIG. 5. The spot shapes of the reference and the signal beam on photo polymer. (a) and (b) are for the reference beam. (c) and (d) are for the signal beam.

and therefore the reference arm is not co-axial. This configuration makes the lens system widely tolerant. The Fourier transform is performed by a three lens system. Its effective focal length is about $85 \mathrm{~mm}$ and the back focal length is about $30 \mathrm{~mm}$. Fig. 4 shows its ray aberration curve which was obtained by using lens simulation software, Code V. The curve shows that the aberration is below $50 \mu \mathrm{m}$. It determines the spot position of the sequential signals on the photo polymer. Since the spot position of the signal beam should be inside the reference beam, the spot size of the reference beam is made larger than that of the signal beam by using the defocusing of lens L3.

Figure 5 shows the profile as measured with the knife edge method of the reference and the signal beam on photo polymer [14]. Dots are measured values and solid lines are Gaussian fitted curves. The $1 / \mathrm{e}^{2}$ beam diameter of the reference beam was measured as $345 \mu \mathrm{m}$ and 389 $\mu m$ in $\mathrm{x}$ and $\mathrm{y}$ directions, respectively. The $1 / \mathrm{e}^{2}$ beam diameter of the signal beam was measured as $129 \mu \mathrm{m}$ and $83 \mu \mathrm{m}$ in $\mathrm{x}$ and $\mathrm{y}$ directions, respectively. 


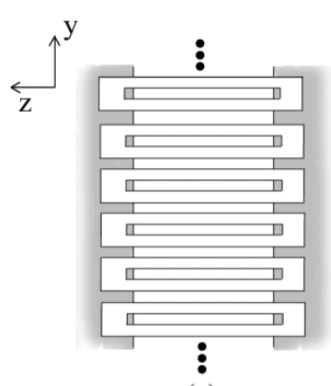

(a)

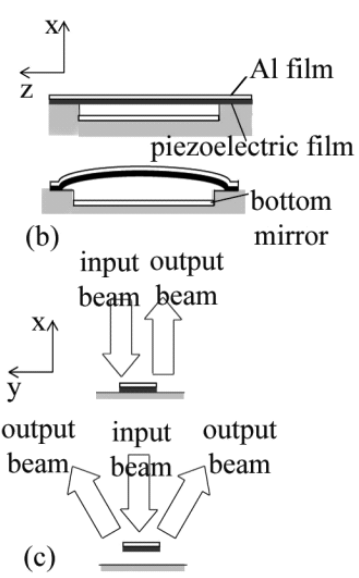

FIG. 6. The simple diagram of our one-dimensional spatial light modulator. (a) Top view, (b) Side view along y-direction, (c) Side view along z-direction.

\section{Spatial light modulator}

Figure 6 shows the one-dimensional spatial light modulator used in our system. Fig. 6(a) is the top view where 480 rectangular ribbons are arranged in the $\mathrm{y}$-direction. The outer length of the ribbon in the $\mathrm{z}$ direction is about $200 \mu \mathrm{m}$ and its outer width in the y direction is about $7.5 \mu \mathrm{m}$. The width of the central hole in a ribbon is about $2.5 \mu \mathrm{m}$ and the space between two ribbons is also about $2.5 \mu \mathrm{m}$. A ribbon consists of a reflective aluminum thin film on top of a piezoelectric film and it hangs over the valley, which is about half the light wavelength deep as shown in Fig. 6(b). The bottom of the valley is flat and coated with reflective aluminum. The top and bottom figures in Fig. 6(b) are side views of a ribbon in the $\mathrm{y}$-direction without and with an applied signal voltage, respectively. As shown in the top of Fig. 6(b), the Al film is flat and parallel to the bottom of the valley when there is no applied voltage. In this case the reflected lights from the ribbon surface and from the bottom of the valley are in phase and the ribbon behaves as a simple mirror. However, when a voltage is applied, the piezoelectric film will extend and be protuberant as much as a quarter of the light wavelength. In this case, the reflected lights from the ribbon surface and the bottom of the valley are out of phase and the ribbon behaves as diffract gratings [15].

\section{TIME SCHEDULING FOR SEQUENTIALSIGNALS AND BRAGG SELECTIVITY OF SEQUENTIAL SUPERIMPOSED HOLOGRAMS}

\section{Time scheduling for sequential signals.}

Generally, the sensitivity of the recording medium and the desired diffraction efficiency of a hologram determine the recording time of the hologram $[16,17]$.

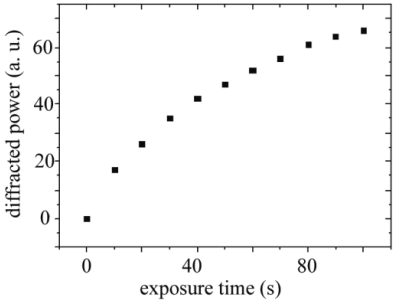

(a)

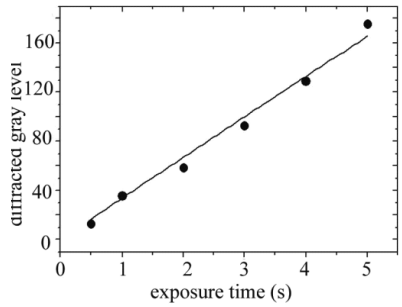

(b)
FIG. 7. The sensitivity of the photopolymer in our experiment (a) in long exposure time, (b) in short exposure time.

Fig. 7 shows the sensitivity of the recording medium used in our experiments. Fig. 7(a) is the diffracted power measured with an optical power meter as a function of the exposure time used in recording a hologram. It shows the nonlinear sensitivity of the recording medium. Fig. 7(b) shows the diffracted power measured with a CCD in a short exposure time below 5 seconds. The dots are measured values of the diffracted power. A nonlinear curve with saturation function,

$$
I_{d}=A[1-\exp (-t / T)]
$$

is fitted to the experimental data, where $I_{d}$ is the gray level of the diffracted peak, $A$ is a saturation value, $t$ is exposure time, and $T$ is time to reach the $63 \%$ of the saturation value.

The diffraction efficiency $\eta$ of a hologram is proportional to the square of the visibility $V$ of the interference fringe formed by the reference and the signal beam [6, 18, 19],

$$
\begin{gathered}
V=\frac{I_{\text {max }}-I_{\text {min }}}{I_{\text {max }}+I_{\text {min }}} \\
\eta \propto V^{2} \propto \frac{\gamma}{(\gamma+1)^{2}}
\end{gathered}
$$

where $I_{\max }$ is the maximum intensity of the fringe, $I_{\min }$ is the minimum intensity of the fringe and $\gamma$ is the ratio of the reference beam intensity $I_{r}$ to the signal beam intensity $I_{s}$. The visibility is only related to the saturation value $A$ in equation (3) because it is independent of the exposure time. Similarly, the saturation time constant $T$ is only related to the recording medium. The visibility may be different for different recorded signals because the position of signal beams on photopolymer may be different.

Fig. 8 shows the measured positions of the signals. These position changes may be from the ray aberration of Fourier lens L1 and the tilt deviation of movable mirror M3. 


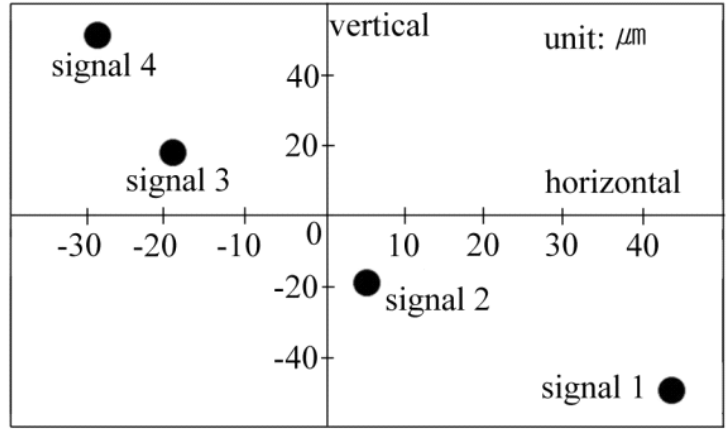

FIG. 8. The position change of signals on photopolymer.

Because the reference beam may not be a perfect plane wave and may not have a Gaussian intensity profile, the position change of the signal beam on the recording medium can cause a change of the fringe visibility. If the visibilities are different for different signal beams, the saturation values of the signals can be different. Therefore, to make the reconstructed signal intensities uniform, we consider the individual sensitivity curves according to the signal sequence numbers. From Eq. (3) the sensitivity curve for the $i$-th signal will be given as $I_{d i}=A_{i}\left[1-\exp \left(t_{i} / t\right)\right]$. In our sequential signal recording, the $i$-th signal will be recorded during the time $\Delta t_{i}=t_{i}-t_{i-1}$. In this case diffracted beam intensity is given by

$$
\Delta I_{d i}=A_{i}\left[1-\exp \left(\Delta t_{i} / T\right)\right]
$$

where $i$ is from 1 to $n$ corresponding to the signal sequence number, $A_{i}$ is the saturation value for the $i$-th signal. For the uniform diffraction intensities the $i$-th diffraction intensity should be same with the first diffraction intensity, $\Delta I_{d 1}=\Delta I_{d i}$, or

$$
A_{1}\left[1-\exp \left(\frac{\Delta t_{1}}{T}\right)\right]=A_{i}\left[1-\exp \left(\frac{\Delta t_{i}}{T}\right)\right]
$$

From the equation we can determine the exposure time $\mathrm{t}_{i}$ as

$$
\Delta t_{i}=T \ln \left\{1-\frac{A_{1}}{A_{i}}\left[1-\exp \left(\frac{\Delta t_{1}}{T}\right)\right]\right\}
$$

The above calculation process is shown schematically in Fig. 9.

To acquire the saturation values for different signals, we recorded holograms with different signals during the same time of 1 second. Fig. 10(a) shows the diffracted signal intensity of the holograms. The ratio between the lowest and the highest signal intensities is about $21 \%$. As can be seen from the figure the first and the

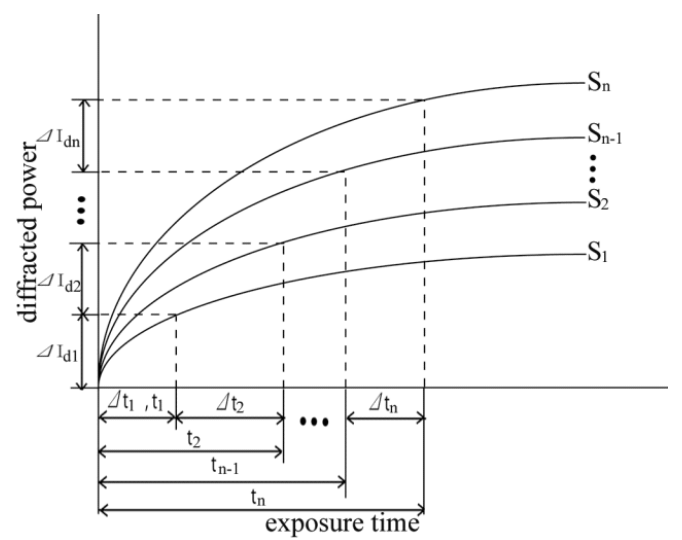

FIG. 9. The sensitivity curves for different signals.

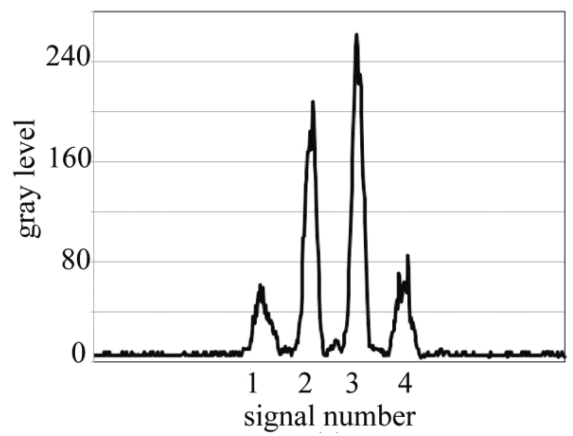

(a)

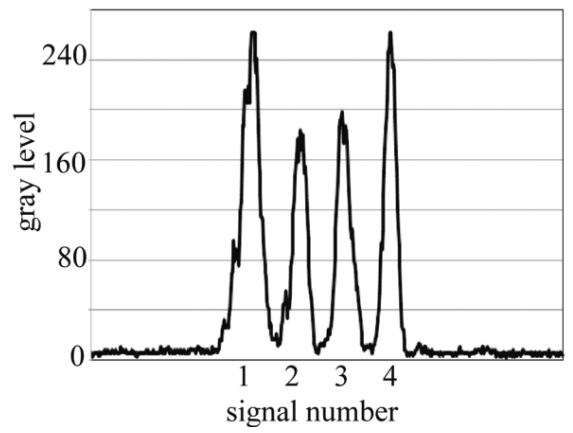

(b)

FIG. 10. Reconstructed signals (a) from holograms with the constant time exposure (b) from holograms with the time scheduling.

fourth reconstructed signals are smaller than the others. It results from the fact that the signal beam position varies due to the ray aberration as shown in Fig. 8 and that the overlap of the first or the fourth signal beam with the reference beam is not as good as that of the other signal beams. We determined the saturation time constants from Fig. 7(b) and the saturation values from Fig. 10(a). Table 1 shows the calculation results by equation (7). We improved on the uniformity of the diffracted signal peaks to about 70\%. Fig. 10(b) shows the time scheduled signals with the exposure times of Table 1. 
TABLE 1. The calculation results from Fig. 7(b) and Fig. 10(a)

\begin{tabular}{l|c|c|c|c}
\hline \hline & Signal 1 & Signal 2 & Signal 3 & Signal 4 \\
\hline $\begin{array}{c}\text { Saturation } \\
\text { value (A) }\end{array}$ & 1230.839 & 4973.594 & 6279.791 & 1607.626 \\
\hline $\begin{array}{c}\text { Saturation } \\
\text { time }(\mathrm{T})\end{array}$ & \multicolumn{4}{|c}{187.4528} \\
\hline $\begin{array}{c}\text { Exposure } \\
\text { time }(\Delta \mathrm{t})\end{array}$ & $1.50 \mathrm{~s}$ & $0.37 \mathrm{~s}$ & $0.29 \mathrm{~s}$ & $1.15 \mathrm{~s}$ \\
\hline
\end{tabular}

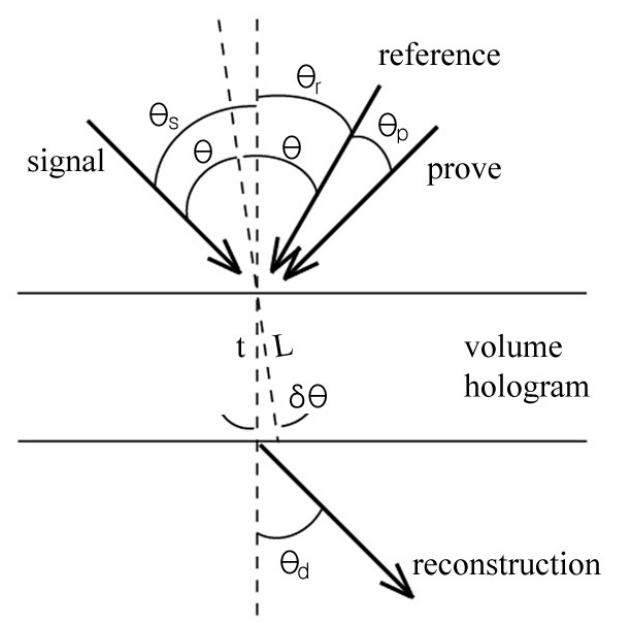

FIG. 11. Schematic geometry for angular Bragg selectivity.

\section{Bragg selectivity of the sequentially recorded hologram}

The holographic storage capacity is strongly connected to the Bragg selectivity. A storage system with smaller Bragg selectivity can have larger storage capacity. In Fig. 11 the reference and signal beams are incident onto a photopolymer of thickness thick . Their incident angles are given by $\theta_{\mathrm{r}}$ and $\theta_{\mathrm{s}}$, respectively. After the hologram is recorded, it is read by the prove beam incident with an angle $\theta_{\mathrm{p}}$.

In this configuration the diffraction efficiency $\eta$ is obtained by the Born approximation [20] as

$$
\eta=\frac{\left(\varepsilon_{1} k t_{\text {thick }}\right)^{2}}{2 \varepsilon_{0} \cos \theta \cos ^{2}(\delta \theta)} \operatorname{sinc}^{2}\left[\frac{2 t_{\text {thick }}(\Delta \theta) \sin \theta}{\lambda \cos (\delta \theta)}\right]
$$

where $\theta=\left(\theta_{s}+\theta_{r}\right) / 2, \delta \theta=\left(\theta_{s}-\theta_{r}\right) / 2, \Delta \theta=\theta_{r}-\theta_{p}$, and $L=t_{\text {thick }} / \cos (\delta \theta)$. Also used in the equation is $\operatorname{sinc}(u)=$ $\sin (\pi u) /(\pi u)$, the dielectric constant $\mathcal{E}_{0}$ of the recording medium, the modulation amplitude of the hologram $\varepsilon_{1}$, and $k=2 \pi / \lambda$. The minimum of the diffraction efficiency occurs at the zero of the sinc function. Therefore angular Bragg selectivity $(\Delta \theta)_{\mathrm{B}}$ is obtained as $(\Delta \theta)_{B}=$ $\lambda \cos (\delta \theta) /\left(2 t_{\text {thick }} \sin \theta\right)$. In our sequential hologram recor- ding, the incident angles of the signal beams are different and therefore the angular Bragg selectivities are different for different signals. When the incident angle of the signal beam is changed by a small amount as $\theta_{\mathrm{s}}=\theta_{\mathrm{so}}+d \theta$, the angular Bragg selectivity is given by

$$
(\Delta \theta)_{B}=\frac{\lambda \cos \left(\frac{\theta_{s o}+d \theta-\theta_{r}}{2}\right)}{2 t_{\text {thick }} \sin \left(\frac{\theta_{s o}+d \theta+\theta_{r}}{2}\right)}
$$

It is noted that the change of the angular Bragg selectivity is negligible for $d \theta \ll \theta_{\text {so }}$ or $\theta_{\mathrm{s}} \ll \theta_{\mathrm{r}}$.

This means that the two-dimensional data page restored from our sequential hologram recording system can have data columns uniformly distributed in the horizontal direction. Similarly, the shift Bragg selectivity can be obtained as

$$
\left(\Delta \delta_{v}\right)_{B} \propto\left(\theta_{s o}+d \theta\right)^{-1} t_{\text {thick }}^{-1}
$$

and the change of the shift Bragg selectivity can also be negligible for $d \theta \ll \theta_{\mathrm{so}}$.

In our experimental condition, $\theta_{\mathrm{so}}$ was $10^{\circ}$ and $d \theta$ was $0.5^{\circ}$, which satisfy the condition of $d \theta \ll \theta_{\text {so }}$ and therefore produce uniformly distributed bit data. We superimposed four holograms sequentially in the experiment. Fig. 12 compares the Bragg selectivity of the sequentially superimposed four holograms with that of a single hologram. Solid squares are the diffracted optical power from the sequentially superimposed holograms. Solid line is the fitting of a sinc function. Open circles are the diffracted optical power from the single hologram. Dash line is the fitting of a sinc function. It can be seen from the figure that there is little change in both the angular and the shift Bragg selectivities for the sequentially superimposed holograms and the single hologram.

Therefore the sequentially superimposed method can increase the storage density. To estimate the storage density, the shift Bragg selectivity was measured by displacing the reference beam and by measuring the diffraction intensity. Then the hologram record area was estimated from the measured shift Bragg selectivity. The storage density for the single exposure hologram was estimated to be about $11 \mathrm{kbit} / \mathrm{mm}^{2}$, which is the ratio of 480 pixels of our modulator to the hologram record area, and that for the sequentially superimposed hologram about $32 \mathrm{kbit} / \mathrm{mm}^{2}$, where four holograms were superimposed. Because we may apply the angle multiplexing seven times in our experimental condition, the maximum storage density is estimated to be $224 \mathrm{kbit} / \mathrm{mm}^{2}$ in our system. 


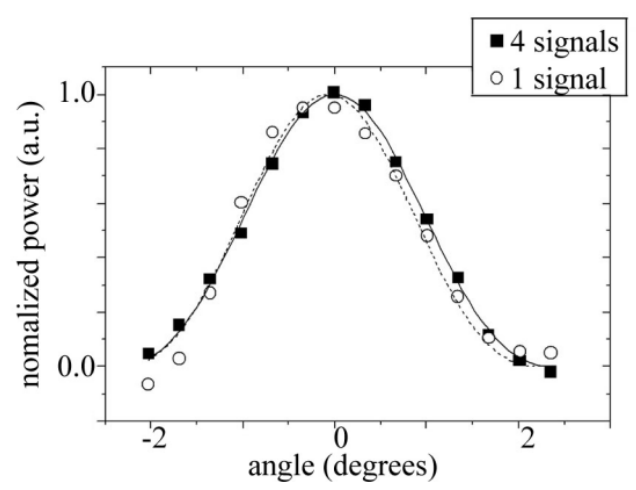

(a)

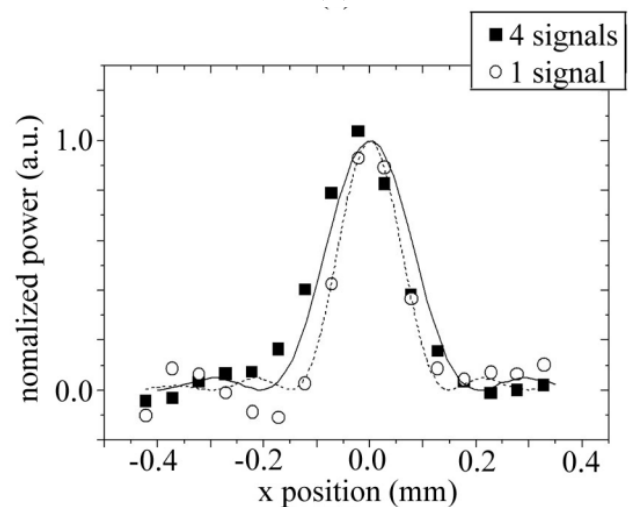

(b)

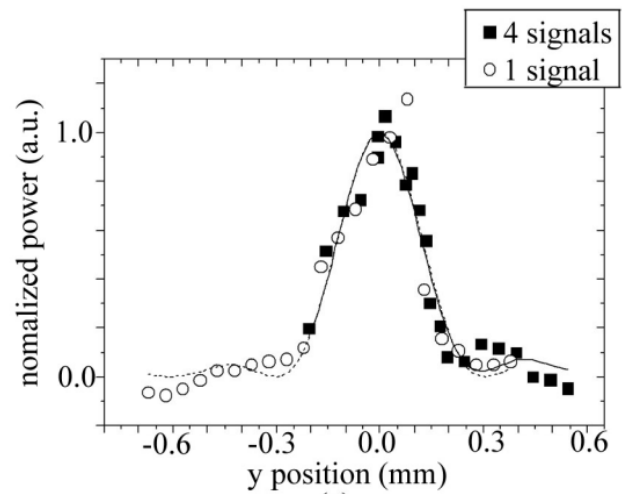

(c)

FIG. 12. Bragg selectivities for sequentially superimposed holograms and a single hologram. (a) Angular Bragg selectivity. (b) Shift Bragg selectivity in x direction. (c) Shift Bragg selectivity in y direction.

\section{CONCLUSION}

We introduced an intermediate holographic storage system using a sequentially superimposed recording technique.

We used an one-dimensional spatial light modulator for high light efficiency and a co-axial lens system for simple system structure. We used a defocusing technique in the reference beam to avoid the deviation of the signal spot position due to the aberration of the conventional lens. Since our method is simple and robust, it can be used to manufacture a holographic storage system cost effectively.

To improve the uniformity of the diffracted signals from the sequentially superimposed holograms, we considered the optical power change of the diffracted signals according to the fringe visibility. In our experiment, we can improve the signal uniformity from $32 \%$ to $70 \%$, which is considered good enough for a digital holographic storage system.

To find the condition for the same Bragg selectivity, we considered the angular Bragg selectivity and the shift Bragg selectivity. The measured Bragg selectivities for the single hologram and for the sequentially superimposed hologram are nearly the same, allowing the increased storage density of our system. The storage density of our system is estimated to be $32 \mathrm{kbit} / \mathrm{mm}^{2}$ without using any multiplexing technique but rather only a line spatial light modulator of 480 pixels.

The storage density of our system may be smaller than that of the conventional holographic storage. However, our system has advantages in the sense that many small packets of data can be recorded sequentially and, later, they can be reconstructed as a larger packet of data. These larger packets of data can be recorded in the conventional holographic storage system. In this case the efficiency of the conventional system can be greatly increased because no small packets of data take a larger block of recording medium.

\section{ACKNOWLEDGMENT}

We thank S. K. Yun and his coworkers of Samsung Electro-Mechanics Co., Ltd. for supporting the spatial light modulator and another apparatus.

\section{REFERENCES}

1. A.-H. Phan, N. Kim, and J.-H. Park, "Input-output coupler system with 45-degree slant angle based on Bragg hologram," J. Opt. Soc. Korea 13, 123-130 (2009).

2. M. Makowski, M. Sypek, I. Ducin, A. Fajst, A. Siemion, J. Suszek, and A. Koloziejczyk, "Experimental evaluation of a full-color compact lensless holographic display," Opt. Exp. 17, 20840-20846 (2009).

3. M.-O. Jeong, N. Kim, and J.-H. Park, "Elemental image synthesis for integral imaging using phase-shifting digital holography," J. Opt. Soc. Korea 12, 275-280 (2008).

4. S. Shin and Y. Yu, "Three-dimensional information and refractive index measurement using a dual wavelength digital holographic microscope," J. Opt. Soc. Korea 13, 173-177 (2009).

5. Q. Weijuan, Y. Yingjie, C. O. Choo, and A. Asundi, "Digital holographic microscopy with physical phase 
compensation," Opt. Lett. 34, 1276-1278 (2009).

6. J. T. Lamachia and C. J. Vincelette, "Comparison of the diffraction efficiency of multiple exposure and single exposure holograms,” Appl. Opt. 7, 1857-1858 (1968).

7. W. J. Carlsen, "Holographic page synthesis for sequential input of data," Appl. Opt. 13, 896-903 (1974).

8. R. K. Kostuk, J. W. Goodman, and L. Hesselink, "Volume reflection holograms with multiple gratings: an experimental and theoretical evaluation," Appl. Opt. 25, 4362-4369 (1986).

9. S. Yasuda, Y. Ogasawara, J. Minabe, K. Kawano, M. Furuki, K. Hayashi, K. Haga, and H. Yoshizawa, "Optical noise reduction by reconstructing positive and negative images from Fourier holograms in coaxial holographic storage systems," Opt. Lett. 31, 1639-1641 (2006).

10. S. Yasuda, K. Kawano, J. Minabe, Y. Ogasawara, K. Hayashi, K. Haga, H. Yoshizawa, and M. Furuki, "Coaxial holographic data storage without recording the dc components," Opt. Lett. 31, 2607-2609 (2006).

11. K. Tanaka, M. Hara, K. Tokuyama, K. Hirooka, K. Ishioka, A. Fukumoto, and K. Watanabe, "Improved performance in coaxial holographic data recording," Opt. Exp. 15, 16196-16209 (2007).

12. S. Yasuda, J. Minabe, and K. Kawano, "Optical noise reduction for dc-removed coaxial holographic data storage,"
Opt. Lett. 32, 160-162 (2007).

13. C. C. Sun, Y. W. Yu, S. C. Hsieh, T. C. Teng, and M. F. Tsai, "Point spread function of a collinear holographic storage system," Opt. Exp. 15, 18111-18118 (2007).

14. G. F. Marshall, Optical Scanning (Marcel Dekker, Inc., New York, USA, 1991), Chapter 1.

15. S. K. Yun, "Spatial optical modulator (SOM): Samsung's light modulator for next-generation laser displays," J. of the SID 15, 321-333 (2007).

16. A. Pu and D. Psaltis, "High-density recording in photopolymer-based holographic three-dimensional disks," Appl. Opt. 35, 2389-2398 (1996).

17. J. T. Sheridan, F. T. O’Neill, and J. V. Kelly, "Holographic data storage: optimized scheduling using the nonlocal polymerization-driven diffusion model," J. Opt. Soc. Am. B 21, 1443-1451 (2004).

18. C. Aleksoff, "Gas lasers as sources for holography," Appl. Opt. 6, 2192-2193 (1967).

19. A. A. Friesem, A. Kozma, and G. F. Adams, "Recording parameters of spatially modulated coherent wavefronts," Appl. Opt. 6, 851-856 (1967).

20. G. Barbastathis and D. Psaltis, "Volume holographic multiplexing methods" in Holographic Data Storage, H. J. Coufal, D. Psaltis, and G. T. Sincerbox, eds. (Springer-V erlag Berlin Heidelbug, New York, USA, 2000). 